\title{
Proceso de rediseño de Maestría en Ingeniería con orientación en Tecnologías de la Información
}

\author{
Aída Lucina González Lara', Claudia García Ancira² \\ Facultad de Ingeniería Mecánica y Eléctrica \\ Universidad Autónoma de Nuevo León \\ Av. Universidad s/n. Ciudad Universitaria. San Nicolás de los Garza, Nuevo León. C.P. 66451 \\ 1'aida.gonzalezlr@uanl.edu.mx, ${ }^{2}$ claudia.garciaa@uanl.mx
}

Fecha de recepción: 16 de agosto 2014

Fecha de aceptación: 2 de octubre 2014

\section{Resumen}

Este trabajo presenta el proceso de rediseño curricular de la Maestría en Ingeniería con orientación en Tecnologías de la Información realizado en la Facultad de Ingeniería Mecánica y Eléctrica (FIME) de la Universidad Autónoma de Nuevo León (UANL). Se siguió el proceso definido por los Lineamientos para Orientar el Proceso de Reforma de los Programas Educativos de Posgrado y acorde con el nuevo Modelo Educativo de la UANL, el cual está sustentado en dos ejes estructuradores, la educación centrada en el aprendizaje y basada en competencias, un eje operativo representado por la flexibilidad de los programas y procesos y dos ejes transversales, innovación académica e internacionalización. Se trabajó de manera integrada y con una base sólida adquirida mediante un diplomado de capacitación, impartido por la Dirección de Estudios de Posgrado, desarrollándose actividades para la generación del perfil de ingreso, de egreso, mapa curricular y competencias generales y específicas.

\section{Palabras Clave}

Rediseño Curricular, Maestría Profesionalizante, Competencias, Tecnologías de la Información. 


\section{Introducción}

Las Maestrías en áreas específicas están orientadas a la formación de recursos humanos y a brindar dominio en campos disciplinarios a través del aprendizaje autónomo y la actitud crítica e innovadora, priorizando el desarrollo profesional.

Con el fin de responder a las necesidades de una nueva sociedad con recursos humanos más especializados y actualizados, acordes al constante desarrollo tecnológico e innovación, y capaces de enfrentar los retos que la globalización impone, se crea en el año 2005 la Maestría en Ingeniería de la Información para formar nuevos profesionistas altamente capacitados en el campo de la Informática, Telemática e Inteligencia Artificial.

En el año 2003 fue creado el programa de Maestría en Ingeniería, el cual cuenta con las orientaciones de Mecánica, Eléctrica, Manufactura, Mecatrónica, y Telecomunicaciones; durante este proceso de rediseño se decide agregar como una nueva orientación llamada Tecnologías de la Información, el rediseño y la actualización de la Maestría en Ingeniería de la Información

\section{Antecedentes}

En 1966 se creó la Escuela de Graduados, antecesora de la actual División de Estudios de Posgrado de la FIME. El posgrado de la FIME se ha estado actualizando continuamente y en este momento ofrece, entre otras opciones, programas de estudio de Maestrías en Ciencias y Maestrías Profesionalizantes.

El objetivo de las maestrías profesionalizantes es formar integralmente profesionales mediante la profundización en el dominio de campos disciplinarios [1]. El programa de Maestría en Ingeniería de la Información ha sido evaluado por organismos externos como CIEES [2] en el 2012, siéndole otorgado el Nivel I, además ha participado en las autoevaluaciones llevadas a cabo para la obtención de los indicadores del PIFI (Programa Integral para el fortalecimiento institucional) todas las recomendaciones han sido tomadas en cuenta en este rediseño.

En la era de la globalización y de los tratados de libre comercio, el sentido de independencia ya no tiene el mismo significado y se orienta a la colaboración internacional. Así, este nuevo enfoque incrementa la necesidad de participación tanto de las Universidades, como de las empresas, en el desarrollo nacional.

Basado en lo anterior la UANL implementa la Visión 2020, que establece: "La Universidad Autónoma de Nuevo León es reconocida en el año 2020 como una institución socialmente responsable y de clase mundial por su calidad, relevancia y contribuciones al desarrollo científico y tecnológico, la innovación, la construcción de escuelas de pensamiento y al desarrollo humano de la sociedad nuevoleonesa y del país" [3].

La UANL define un nuevo Modelo Educativo UANL [1] el cual está basado en competencias y se sustenta en dos ejes estructuradores, la educación centrada en el aprendizaje y basada en competencias, un eje operativo donde se refleja la flexibilidad 
de los programas y procesos educativos y dos ejes transversales, la innovación académica y la internacionalización como se muestra en la Figura 1.

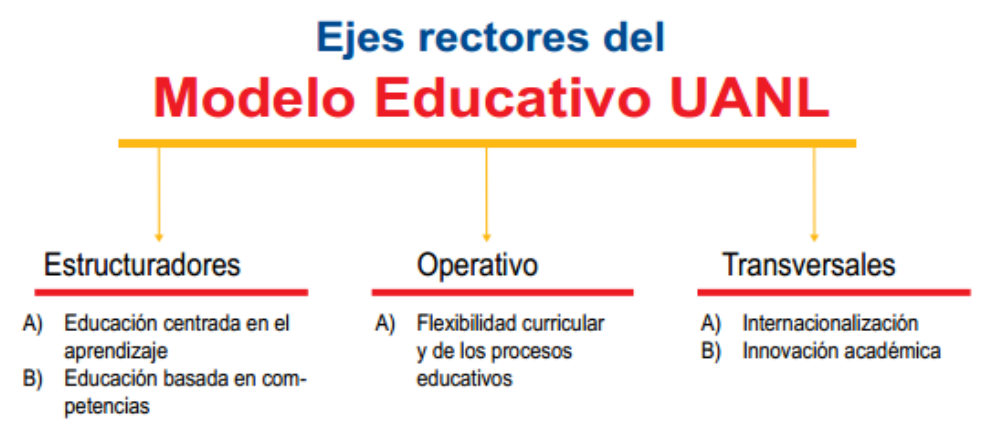

Fig. 1. Esquema de los ejes rectores del Modelo UANL.

\section{Metodología}

De manera inicial, para llevar a cabo el proceso de actualización y rediseño de la Maestría en Ingeniería con orientación en Tecnologías de la Información (MITI) se atendió la invitación de la Dirección de Estudios de Posgrado de la UANL al Diplomado en Procesos de Diseño e Implementación Curricular para el Modelo Educativo que se cursa en modalidad semipresencial. En este diplomado se desarrollan temas que apoyan a los responsables de los programas en el rediseño de los mismos; las unidades de aprendizaje de dicho Diplomado son: Innovación y cambio en el campo curricular, Diseño curricular, Facilitación y evaluación del aprendizaje y competencias y Seguimiento y evaluación curricular.

A continuación se enumeran algunas de las principales acciones llevadas a cabo durante este proceso.

Juntas de Trabajo. La juntas de trabajo semanales del Coordinador con los profesores de su Núcleo Básico Académico fueron actividades básicas para lograr el objetivo ya que el trabajo colaborativo permitió la adecuada comunicación y enriquecimiento de los Programas Educativos.

Uso de herramientas de apoyo en el proceso. Se proporcionó una plantilla de Lineamientos para Orientar el Proceso de Reforma de los Programas Educativos de Posgrado (LOPREP) [4] que contiene la guía de para la estructuración del documento de Propuesta para el rediseño del programa, además se implementó una plataforma web Ilamada Sistema de Evaluación de Programas de Posgrado, [5] en dicha plataforma se sube el documento de la Propuesta de rediseño para que sea evaluado por los profesores asignados como evaluadores de los programas rediseñados, una vez evaluado el documento se publica un dictamen en el que se hacen recomendaciones y los responsables del programa atienden estas recomendaciones, 
de manera iterativa se realiza este proceso hasta que la evaluación de la propuesta sea satisfactoria.

Implementación del Modelo Educativo UANL. Los ejes estructuradores del Modelo educativo UANL dan estructura a los programas educativos, pues su diseño requiere considerar al estudiante como centro del proceso para promover un aprendizaje significativo; de igual forma, permiten reconocer que el proceso educativo integral implica la adquisición de competencias, entendidas como la expresión concreta del conjunto de conocimientos, habilidades, destrezas, actitudes y valores, que pone en juego la persona cuando lleva a cabo una actividad [1] El eje rector educación centrada en el aprendizaje se orienta a lograr aprendizajes significativos utilizando estrategias cognitivas del pensamiento lógico y creativo, promoviendo la actividad autónoma del estudiante mediante una participación más activa y responsable en la construcción de su propio conocimiento. Los Criterios que marcan este eje son: aprendizaje autónomo, aprendizaje significativo, aproximación al conocimiento, aprendizaje constructivo, tutoría y autoconfianza

El eje rector de educación basada en competencias busca ligar la escuela con el mundo de la vida y del trabajo e impacta en la operación del programa, para lo cual se desarrollan los siguientes apartados: Perfil de Egreso, Competencias generales, Competencias del programa, Niveles de dominio de las competencias específicas del programa educativo y Tabla de congruencia de unidades de aprendizaje con las competencias generales y específicas, Relación de unidades de aprendizaje con niveles de dominio de las competencias específicas, Integración de las unidades de aprendizaje con clave, créditos, horas, y requisito, Distribución curricular y Producto integrador.

\section{Resultados}

\subsection{Perfil de Egreso}

Para esta tarea se realizaron encuestas con egresados, alumnos y empresarios del área para identificar las funciones y actividades que debe realizar el egresado en épocas actuales y se investigó acerca de las características del contexto nacional e internacional así como las ofertas educativas de otras instituciones.

La Maestría en Ingeniería con orientación en Tecnologías de la Información proporcionará a los egresados una preparación de alto nivel que los capacitará con las habilidades y conocimientos suficientes para:

- Desarrollar soluciones innovadoras de ingeniería en el área de Tecnologías de la Información y la Comunicación.

- Administrar proyectos de Tecnologías de la Información y la comunicación.

- Analizar, interpretar y generar soluciones a los problemas de la Industria que afectan a la empresa en el entorno de la Tecnología de Información y la 
Comunicación con un sentido ético, a través de la autocrítica, la creatividad y la disciplina.

\subsection{Competencias Generales}

El Modelo Educativo de la UANL comprende 15 competencias generales clasificadas en tres categorías, estas son: [1]

\section{Competencias instrumentales}

Estas competencias tienen una función instrumental y pueden ser de naturaleza lingüística, metodológica, tecnológica o cognoscitiva, propias del perfil académico y profesional necesario para la competitividad local e internacional en la época actual.

\section{Competencias personales y de interacción social}

Son las competencias que facilitan el proceso de desarrollo humano personal e interpersonal, es decir, la interacción social y cooperación a través de la expresión de sentimientos, la crítica y la autocrítica.

\section{Competencias integradoras}

Este tipo de competencias integran las competencias instrumentales con las personales y de interacción social, para que el egresado alcance, junto al desarrollo de las competencias específicas, la formación integral que lo haga competitivo, tanto a nivel local, como nacional e internacional.

El cumplimiento de las competencias generales se logra incluyendo en las unidades de aprendizaje al menos una de las competencias generales [4].

\subsection{Competencias Específicas}

En la propuesta del rediseño del programa educativo se definen competencias específicas, todas las unidades de aprendizaje deberán contener al menos una de estas competencias específicas.

La Tabla 1 muestra las competencias específicas de la MITI.

\begin{tabular}{|c|c|}
\hline $\begin{array}{l}\text { COMPETENCIAS } \\
\text { ESPECIFICAS }\end{array}$ & DECLARACIÓN \\
\hline CEITI1 & $\begin{array}{l}\text { Analiza los procesos de negocio del entorno de Tecnologías de la Información y } \\
\text { la Comunicación con la finalidad de diseñar y desarrollar soluciones de calidad. } \\
\text { Identifica oportunidades de mejora en los procesos de negocio para crear } \\
\text { soluciones innovadoras mediante la integración de las herramientas y estrategias } \\
\text { de Tecnologías de la Información y la Comunicación. }\end{array}$ \\
\hline CEITI3 & $\begin{array}{l}\text { Administra proyectos de Tecnologías de la Información y la Comunicación } \\
\text { mediante la aplicación de metodologías y de mejores prácticas con la finalidad } \\
\text { de optimizar los procesos de negocio. }\end{array}$ \\
\hline CEITI4 & $\begin{array}{l}\text { Estructura conocimiento mediante el análisis de datos de la organización con la } \\
\text { finalidad de apoyar en forma efectiva en la toma de decisiones para mejorar la } \\
\text { operación del negocio en un entorno competitivo y globalizado. }\end{array}$ \\
\hline
\end{tabular}

Tabla 1. Muestra las competencias específicas de la MITI. 
Cada una de las competencias específicas se desglosan en niveles de dominio, este nivel de dominio expresa los niveles de desarrollo de las competencias que posteriormente definirán este nivel en cada unidad de aprendizaje, el formato para determinar los niveles de dominio de las competencias específicas se ejemplifica en la Tabla 2.

La representación de la relación de cada unidad de aprendizaje con las competencias tanto generales como específicas con las que está vinculada permite visualizar esta relación así como la comprobación de que todas las competencias estén incluidas en las unidades de aprendizaje, el formato utilizado se muestra en la Tabla 3.

\begin{tabular}{|c|c|c|c|c|}
\hline $\begin{array}{l}\text { Competencia } \\
\text { Especifica }\end{array}$ & $\begin{array}{c}\text { Nivel I } \\
\text { Inicial }\end{array}$ & $\begin{array}{l}\text { Nivel II } \\
\text { Básico }\end{array}$ & $\begin{array}{l}\text { Nivel III } \\
\text { Autónomo }\end{array}$ & $\begin{array}{l}\text { Nivel IV } \\
\text { Estratégico }\end{array}$ \\
\hline CEITI1 & $\begin{array}{l}\text { Identifica las } \\
\text { principales } \\
\text { metodologías para el } \\
\text { análisis de los } \\
\text { procesos de negocio }\end{array}$ & $\begin{array}{ll}\text { Aprende } & \text { la } \\
\text { utilización } & \text { de } \\
\text { metodologías } & \end{array}$ & $\begin{array}{lr}\text { Aplica } & \text { las } \\
\text { metodologías para } \\
\text { el diseño de } & \text { los } \\
\text { procesos } & \text { de } \\
\text { negocio } & \\
\end{array}$ & $\begin{array}{l}\text { Desarrolla } \\
\text { soluciones de } \\
\text { calidad para los } \\
\text { procesos de negocio }\end{array}$ \\
\hline CEITI2 & $\begin{array}{l}\text { Genera } \\
\text { oportunidades de } \\
\text { mejora a partir de } \\
\text { las necesidades del } \\
\text { cliente. }\end{array}$ & $\begin{array}{lr}\text { Integra } & \text { las } \\
\text { herramientas } & \text { y } \\
\text { estrategias } & \text { de } \\
\text { tecnologías de } & \text { la } \\
\text { información } & y \\
\text { comunicaciones } & \\
\text { para } & \text { la } \\
\text { construcción } & \text { de } \\
\text { soluciones } & \\
\end{array}$ & $\begin{array}{lr}\text { Manipula la } \\
\text { información para } \\
\text { la propuesta de } \\
\text { soluciones }\end{array}$ & $\begin{array}{l}\text { Diseña soluciones } \\
\text { innovadoras }\end{array}$ \\
\hline CEITI3 & $\begin{array}{lrr}\text { Reconoce } & & \text { los } \\
\text { recursos } & \text { que } & \text { se } \\
\text { utilizarán } & \text { en } & \text { el } \\
\text { proyecto } & & \\
\end{array}$ & $\begin{array}{ll}\text { Administra } & \text { los } \\
\text { recursos } & \text { del } \\
\text { proyecto } & \end{array}$ & 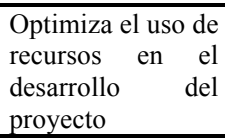 & $\begin{array}{l}\text { Implementa las } \\
\text { soluciones para la } \\
\text { mejora continua }\end{array}$ \\
\hline CEITI4. & $\begin{array}{lr}\text { Revisa } & \text { los } \\
\text { fundamentos para la } \\
\text { construcción de } & \text { los } \\
\text { objetos } & \text { de } \\
\text { información } & \end{array}$ & $\begin{array}{lr}\begin{array}{l}\text { Investiga } \\
\text { objetos }\end{array} & \begin{array}{r}\text { los } \\
\text { de }\end{array} \\
\text { información } & \text { que } \\
\text { son cruciales } & \text { para } \\
\text { la toma de } & \text { decisiones } \\
\end{array}$ & $\begin{array}{lr}\text { Evalúa } & \text { los } \\
\text { objetos } & \text { de } \\
\text { información } & \end{array}$ & $\begin{array}{l}\text { Propone soluciones } \\
\text { de mejora para la } \\
\text { toma de decisiones }\end{array}$ \\
\hline
\end{tabular}

Tabla 2. Muestra el desglose de niveles de dominio de las competencias específicas.

\begin{tabular}{|c|c|c|c|c|c|c|c|c|c|c|c|c|c|c|c|c|c|c|c|c|}
\hline \multirow{3}{*}{ Clave } & \multirow{3}{*}{$\begin{array}{l}\text { Unidad de } \\
\text { aprendizaje }\end{array}$} & \multicolumn{15}{|c|}{ Competencias generales } & \multicolumn{4}{|c|}{$\begin{array}{l}\text { Competencias } \\
\text { específicas }\end{array}$} \\
\hline & & \multicolumn{8}{|c|}{ Instrumentales } & \multicolumn{4}{|c|}{$\begin{array}{l}\text { Personales y de } \\
\text { interacción } \\
\text { social }\end{array}$} & \multicolumn{3}{|c|}{ Integradoras } & \multicolumn{4}{|c|}{$\begin{array}{c}\text { Tecnologías de } \\
\text { la Información } \\
\text { CEITI }\end{array}$} \\
\hline & & 1 & 2 & 3 & 4 & 5 & 6 & 7 & 8 & 9 & 10 & 11 & 12 & 13 & 14 & 15 & 1 & 2 & 3 & 4 \\
\hline MITIBA001 & $\begin{array}{l}\text { Matemáticas para } \\
\text { ingeniería }\end{array}$ & & $\bar{X}$ & $\mathrm{X}$ & & & & & & & & & & & & & $\mathrm{X}$ & $\mathrm{X}$ & & \\
\hline MITIBA002 & $\begin{array}{l}\text { Investigación para } \\
\text { Ingeniería }\end{array}$ & & $\mathrm{X}$ & & & & & & $\bar{X}$ & & & & & & & & $\mathrm{X}$ & $\mathrm{X}$ & & \\
\hline MITIAV001 & $\begin{array}{ll}\text { Ingeniería } & \text { de } \\
\text { software } & \end{array}$ & & & & & $\mathrm{X}$ & & & & & & & & & & & $\mathrm{X}$ & $\mathrm{X}$ & $\mathrm{X}$ & \\
\hline
\end{tabular}

Tabla 3. Muestra la relación de unidades de aprendizaje con las competencias generales y específicas. 


\subsection{Distribución curricular}

Una actividad crucial en este proceso está representada por la definición del mapa curricular, de acuerdo a la definición del Modelo Académico de Posgrado, el programa debe de tener materias de Formación Básica, Formación Avanzada, de Aplicación, Profesionalizante, Libre Elección y un Producto Integrador [6].

El área curricular de formación básica proporciona la comprensión de las unidades de aprendizaje fundamentales que se requieren en el programa educativo de maestría. En el área de formación avanzada se proponen las unidades de aprendizaje que contienen los fundamentos teóricos más avanzados en el área disciplinar correspondiente. El área de aplicación corresponde a las unidades de aprendizaje, laboratorios y prácticas que manejan los avances tecnológicos y de aplicación. El área curricular de libre elección tiene como objetivo que el estudiante pueda seleccionar unidades de aprendizaje del conjunto de la oferta educativa de posgrado de la Universidad y de otras universidades con las que exista cooperación académica, con el objeto de fortalecer sus competencias y su formación integral. En el área curricular profesionalizante, se ofrecen unidades de aprendizaje que tienen como finalidad formar integralmente profesionales mediante la profundización en el dominio de campos disciplinarios a través del aprendizaje autónomo y la actitud crítica e innovadora [6].

Se definió que la cantidad de créditos para las áreas de Formación Básica, Formación Avanzada y de Aplicación sea de 16, para el área profesioanlizante se definieron 17 créditos, para libre elección 4 créditos y el producto integrador será de 11 créditos.

La Maestría en Ingeniería con orientación en Tecnologías de la Información consta de un total de 80 créditos distribuidos como se muestra en la Figura 2.

El producto integrador consiste en el desarrollo de un proyecto ingenieril aplicando el método científico para solucionar problemas del entorno productivo, comercial, de servicios o educativo.

De acuerdo al proyecto educativo, los estudiantes podrán graduarse en un tiempo de 2 años (6 tetramestres), considerando que el alumno curse dos unidades de aprendizaje por tetramestre. 


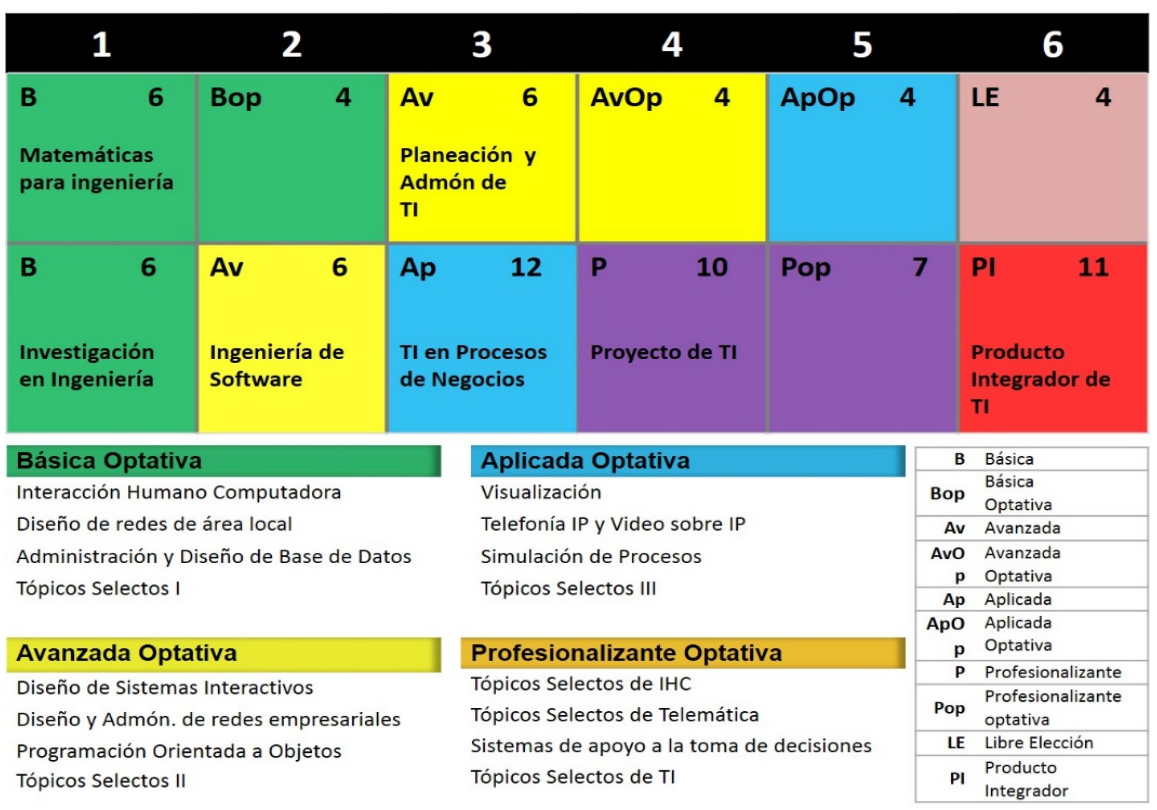

Fig. 2. Representación de la distribución de unidades de aprendizaje y créditos de la MITI.

\section{Conclusiones}

El uso de TIC contribuye al crecimiento económico y genera empleos como resultado de mejoras en la productividad de las empresas y la creación de nuevas industrias, las TIC dan un fuerte impulso para la adopción de nuevos métodos más eficientes de producción, asimismo, la digitalización está altamente relacionada con la innovación y también fomenta el uso más inteligente y ecológico de los recursos humanos, naturales y económicos, debido a lo anterior es de suma importancia que los programas relacionados con Tecnologías de Información estén actualizados. El proceso de rediseño del programa educativo requirió el trabajo colaborativo entre varios departamentos de la FIME incluyendo subdirectores, coordinadores y profesores quienes apoyaron en la recopilación de información, investigaciones y entrevistas, lo que enriqueció el producto final ya que se conjuntaron ideas y experiencias de los implicados en el proceso. La planeación del proceso así como la orientación por parte de la Dirección de Estudios de Posgrado permitieron el logro del objetivo. El apoyo y colaboración de organismos como MexicolT, Prosoft y el Clúster de Software de Nuevo León contribuyen a la actualización de los programas educativos mediante la vinculación constante entre las personas involucradas de las instituciones y de la academia. Se trabaja en la implementación de este rediseño con la capacitación de los profesores mediante un Diplomado de Docencia impartido por la Dirección de Estudios de Posgrado, en dicho Diplomado se desarrollan los programas 
analíticos de las unidades de aprendizaje definidas para el programa de maestría. Se dará seguimiento al proceso de implementación para evaluar los resultados obtenidos

\section{Referencias}

[1] Universidad Autónoma de Nuevo León, «Modelo Educativo de la UANL,» 18 Junio 2008. [En línea]. Disponible:

http://www.uanl.mx/sites/default/files/dependencias/del/mod-educativo-08web.pdf.

[2] CIEES, «Comités Interinstitucionales para la Evaluación de la Educación Superior, A.C., 7 Marzo 2013. [En línea].

Disponible: www.ciees.edu.mx/ciees/inicio.php.

[3] UANL, «Visión 2020 UANL,» 15 Octubre 2011. [En línea]. Disponible:

http://www.uanl.mx/sites/default/files/documentos/universidad/folletovision2020uanl.pdf.

[4] D. d. E. d. Posgrado, «Lineamientos para Orientar el Proceso de Reforma de los Programas Educativos de Posgrado,» Monterrey, 2011.

[5] DGI UANL, «Sistema de Evaluación de Posgrado,» 10 Enero 2012. [En línea]. Disponible: http://www.evaluacionposgrado.uanl.mx.

[6] Secretaría de Investigación, Innovación y Posgrado, «UANL,» 01 Junio 2011. [En línea]. Disponible:

http://www.uanl.mx/sites/default/files/Modelo.Academico.posgrado.pdf 